\title{
Development and validation of nomogram to predict very early recurrence of combined hepatocellular-cholangiocarcinoma after hepatic resection: a multi-institutional study
}

Yijun Wu ${ }^{1 \dagger}$, Hongzhi Liu ${ }^{1 \dagger}$, Jianxing Zeng ${ }^{1 \dagger}$, Yifan Chen ${ }^{2 \dagger}$, Guoxu Fang ${ }^{1}$, Jinyu Zhang ${ }^{1}$, Weiping Zhou ${ }^{3}$, Yongyi Zeng ${ }^{1 *}$ and Jingfeng Liu $^{4,5^{*}}$

\begin{abstract}
Background and objectives: Combined hepatocellular cholangiocarcinoma (CHCC) has a high incidence of early recurrence. The objective of this study is to construct a model predicting very early recurrence (VER) (i.e., recurrence within 6 months after surgery) of CHCC.

Methods: One hundred thirty-one consecutive patients from Eastern Hepatobiliary Surgery Hospital served as a development cohort to construct a nomogram predicting VER by using multi-variable logistic regression analysis. The model was internally and externally validated in a validation cohort of 90 patients from Mengchao Hepatobiliary Hospital using the C concordance statistic, calibration analysis, and decision curve analysis (DCA).

Results: The VER nomogram contains microvascular invasion (MiVI), macrovascular invasion (MaVl), and CA19-9 $>25 \mathrm{mAU} / \mathrm{mL}$. The model shows good discrimination with C-indexes of $0.77(95 \% \mathrm{Cl}: 0.69-0.85)$ and 0.76 (95\% Cl: $0.66-0.86$ ) in the development cohort and validation cohort respectively. Decision curve analysis demonstrated that the model is clinically useful and the calibration of our model was favorable. Our model stratified patients into two different risk groups, which exhibited significantly different VER.
\end{abstract}

Conclusions: Our model demonstrated favorable performance in predicting VER in CHCC patients.

Keywords: Combined hepatocellular-cholangiocarcinoma (cHCC), Very early recurrence, Nomogram, Prognosis

*Correspondence: lamp197311@126.com; drjingfeng@126.com

${ }^{\dagger}$ Yijun Wu, Hongzhi Liu, Jianxing Zeng and Yifan Chen contributed equally to this work.

1 Department of Hepatobiliary Surgery, Mengchao Hepatobiliary Hospital of Fujian Medical University, Fuzhou 350025, People's Republic of China

${ }^{5}$ The Big Data Institute of Southeast Hepatobiliary Health Information,

Mengchao Hepatobiliary Hospital of Fujian Medical University, Fuzhou 350025, People's Republic of China

Full list of author information is available at the end of the article

\section{Introduction}

Combined hepatocellular cholangiocarcinoma (cHCC) is a rare subtype, accounting for only $0.4-14.2 \%$ of primary liver carcinoma (PLC) [1-5]. Due to its rarity and a wide variety of pathological types, the research in $\mathrm{cHCC}$ has been tough and the clinicopathological characteristics and prognosis of $\mathrm{cHCC}$ still remain poorly understood.

It is well-acknowledged that the prognosis of $\mathrm{cHCC}$ is dismal, due to its high recurrence rate after hepatic resection [2]. On the basis of previous studies [1-5], original author(s) and the source, provide a link to the Creative Commons licence, and indicate if changes were made. The images or other third party material in this article are included in the article's Creative Commons licence, unless indicated otherwise in a credit line to the material. If material is not included in the article's Creative Commons licence and your intended use is not permitted by statutory regulation or exceeds the permitted use, you will need to obtain permission directly from the copyright holder. To view a copy of this licence, visit http://creativecommons.org/licenses/by/4.0/. The Creative Commons Public Domain Dedication waiver (http://creativeco mmons.org/publicdomain/zero/1.0/) applies to the data made available in this article, unless otherwise stated in a credit line to the data. 
most recurrences occur early after the hepatic resection for $\mathrm{CHCC}$. Patients with $\mathrm{cHCC}$ suffered early recurrence (ER) rate of $57-75 \%$ and 6 months recurrence rate of about $40 \%$ or so [4-9], higher than patients with HCC, similar to patients with ICC $[2,10]$.

Nevertheless, most studies only concentrated on ER for patients with cHCC using a cut-off of 2 years, which consists with the cut-off for ER of hepatocellular carcinoma (HCC) and intrahepatic cholangiocarcinoma (ICC) [4-8]. The ER rate and the relationship between ER and clinicopathological characteristics of cHCC have been researched in these studies [4-8]. Given that patients with $\mathrm{cHCC}$ suffered earlier recurrence and a higher rate of recurrence than patients with $\mathrm{HCC}[2,3]$, it may not be appropriate to use a cut-off of 2 years to differentiate early and late recurrence for patients with $\mathrm{cHCC}$ or ICC [11], aligning with patients with HCC.

In the field of other malignant tumors [11-13], including HCC and ICC [11, 13], researchers have noticed that patients who had VER(i.e., recurrence within 6 months after surgery) suffered a significant lower OS than those who did not have VER, and therefore brought forward the definition of VER and investigated the risk factors of VER. The research on VER would be of significance for patients with cHCC. As such, the tool we developed to predict VER in patients with cHCC after hepatic resection would help identify the patients at high risk for VER, therefore making a contribution to constructing individualized surveillance strategies following hepatic resection or recommending treatment postoperatively.

To the best of our knowledge, no study exists has investigated the prediction of VER of cHCC after surgical resection. Therefore, our study aims to illustrate the relationship between the clinicopathological characteristics and VER, develop, and validate a model predicting VER after hepatic resection for $\mathrm{cHCC}$ based on multi-institutional data sets. In addition, we have constructed an online calculator to promote the use of our model in clinical work (https:// chcrecurrence.shinyapps.io/myCHCVER2).

\section{Patients and methods}

The analysis was presented in accordance with the TRIPOD (Transparent reporting of a multi-variable prediction model for individual prognosis or diagnosis) guidelines [14].

\section{Patients}

In this retrospective multi-institutional study, a total of 221 patients received hepatic resection for cHCC between January 2011 and December 2015 were enrolled from 2 institutions, compromising Eastern Hepatobiliary Surgery Hospital of Second Military Medical University (Institution I) and Mengchao Hepatobiliary Hospital of
Fujian Medical University (Institution II). This study was conducted in accordance with the ethical guideline of the 1975 Declaration of Helsinki and obtained approval from the Institutional Ethics Committee of the Mengchao Hepatobiliary Hospital of Fujian Medical University. Informed consent has been given to each participants enrolled in this study.

The inclusion criteria include (1) patients underwent curative hepatic resection for primary $\mathrm{cHCC}$ and pathologically confirmed as cHCC (only Allen and Lisa classification type C ); (2) Child-Pugh A or B liver function; (3) no previous anti-tumor treatment; (4) no extrahepatic metastasis; and (5) R0 resection, defined as complete removal of macroscopic tumor nodules with a clear margin. The exclusion criteria include (1) other malignant tumors; (2) incomplete clinical data; and (3) loss to follow-up within 12 months after the surgery.

One hundred thirty-one eligible patients form Institution I were assigned to development cohort for the construction of the predictive model and 90 eligible patients form Institution II were allocated to validation cohort for the verification of the models. The flow chart of this study was shown in Fig. 1.

\section{Hepatic resection}

All patients underwent hepatic resection, the type of which was determined by the tumor size, tumor location, preoperative diagnosis, and liver function. Hepatic resection without lymph node dissection was performed to the patients who were diagnosed as HCC preoperatively. Hepatic resection with lymph node dissection was performed to the patients who were diagnosed as ICC or cHCC preoperatively. Lymph node dissection was performed when an abnormal swollen lymph node around the hepatoduodenal ligament, the common hepatic artery, or behind the pancreas head was seen intraoperatively. Major resection was defined as resection of 3 or more Couinaud segments, while minor resection was defined as resection of fewer than 3 Couinaud segments [15].

\section{Clinicopathological characteristics and definitions}

The clinicopathological variables were listed as follows: gender, age, hepatitis B virus (HBV), hepatitis C virus (HBV), non-alcoholic fatty liver disease(NAFLD), red blood cell count, hemoglobin, platelet count, neutrophil count, lymphocyte count, neutrophils/lymphocytes ratio , aspartate aminotransferase, prothrombin time, total bilirubin, albumin, gamma-glutamyl transpeptidase, alkaline phosphatase, alpha-fetoprotein, carbohydrate antigen 19-9 level, carcinoembryonic antigen, decarboxylic prothrombin, liver cirrhosis, resection type, lymph node dissection, tumor number, maximum tumor size, 
Pathology confirmed cHCC patients who underwent hepatic resection between 2011/1/1 and 2015/12/31 from two institution ( $n=363$ )

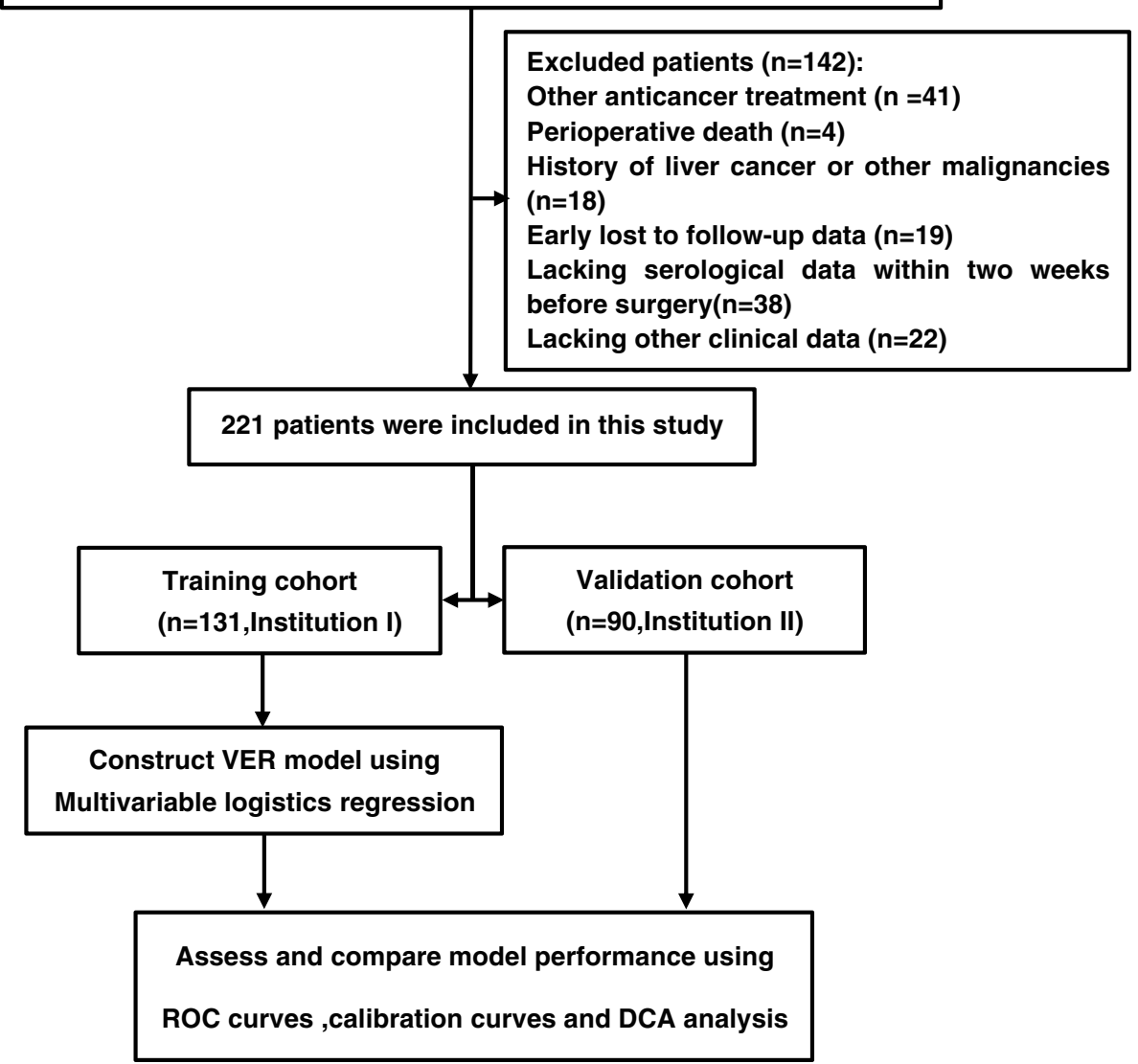

Fig. 1 The flow chart of this study

tumor capsule, microvascular invasion (MiVI), macrovascular invasion(MaVI), satellite nodules (SN), Edmondson-Steiner classification [16], intraoperative blood loss, intraoperative blood transfusion, Child-Pugh classification, and AJCC 8th edition staging manual. We collected the serological examinations carried out within 2 weeks before the surgery. Two independent pathologists majored in hepatic tumors reviewed the resected specimens. MiVI was defined as the presence of tumor cell nests in the portal vein, the hepatic vein or the capsular vessel lined by endothelium visible only under microscopy [17], MaVI was defined as invasion of the firstand second- order branches of the portal veins, hepatic arteries or hepatic veins [18], SN was defined as tumor cell nests present on microscopy or tumors of which the maximum diameter is less than $2 \mathrm{~cm}$ resenting within 2 $\mathrm{cm}$ of the main tumor on macroscopy [19]. Tumor stage was defined in accordance with the AJCC 8th edition ICC staging manual [20].

\section{Follow-up}

After the surgery, patients were followed up every 3 months in the first 2 years and every 6-12 months thereafter. Serum tumor markers, contrast-enhanced magnetic resonance imaging (MRI) of abdomen were performed to diagnose the recurrence of $\mathrm{cHCC}$. Patients with recurrence of $\mathrm{cHCC}$ received appropriate treatments including transarterial chemoembolization (TACE), radiofrequency ablation or hepatic re-resection. As the primary end point, recurrence-free survival (RFS) was defined as the interval from hepatic resection to recurrence. VER of $\mathrm{cHCC}$ was defined as recurrence within 6 months after surgery on the basis of previous studies [11-13, 21]. We collected the survival information until March 31, 2020.

\section{Statistical analysis}

Categorical variables were presented as number (percentage) and compared using the chi-square test or Fisher exact test. Normally distributed continuous 
variables were presented as mean (standard deviation, IQR) and compared using Mann-Whitney $U$ test. The $P$ value less than 0.05 was set as statistical significance in this study. Statistical analysis was conducted by using $R$ version 4.1.0 (http://www.r-project.org/) with $R$ packages of Table 1, rms, pROC, ggplot2, shiny, survminer, and survival.

The optimal thresholds of continuous variables were determined by analyzing the ROC curves and Youden index. Clinicopathological variables showing potentially relevance (with $p<0.05$ in the univariable logistic regression analysis) were employed for the multi-variable logistic regression analysis. The prognostic factors determined by using a stepwise selection method were integrated to construct the VER model. Nomogram and an online calculator are built to facilitate the clinical use of our model [22] (https://chcrecurrence.shinyapps.io/myCHCVER2). The VER risk scores of patients were calculated in accordance with the VER model, then the probabilities of VER were estimated by using the formula: VER probability $=1 /$ $\{1+\exp [-($ VER risk score $)]\}$. All patients were stratified into two risk groups depending on their VER probability by the cut-offs of 50th centile. Kaplan-Meier curves and log-rank test were employed to evaluate the difference in RFS between different subgroups.

To assess the predictive accuracy of our model, we drew the ROC curves and calculated the $\mathrm{C}$-indexes of our model and AJCC staging for the development cohort and validation cohort with the bootstrapping resample method $(n=1000)$. We conducted the decision curve analysis(DCA) to estimate the clinical net benefits of our model and AJCC staging for both cohorts with the bootstrapping resample method $(n=1000)$ [23]. Calibration curves were employed to plot the predicted probabilities verses the actual outcomes.

\section{Results}

\section{Clinicopathologic characteristics}

A total of $363 \mathrm{cHCC}$ patients underwent hepatic section in Eastern Hepatobiliary Surgery Hospital of Second Military Medical University (Institution I) and Mengchao Hepatobiliary Hospital of Fujian Medical University (Institution II) between 1 January 2011 and 31 December 2015 received careful reviews of their medical records, 221 patients were eligible for the study, 142 patients were excluded for receiving other anti-cancer treatment $(n=41)$, perioperative death $(n=4)$, history of liver cancer or other malignancies $(n=18)$, early lost to follow-up data $(n=19)$, lacking serological data within 2 weeks before surgery ( $n$ $=38)$, lacking other clinical data $(n=22)$. One hundred thirty-one eligible patients from Institution I were enrolled as the development cohort and 90 eligible patients from Institution II serve as the validation cohort. The flow chart of this study is shown in Fig. 1.

The clinicopathologic characteristics of patients in the development cohort and the validation cohort are summarized in Table 1. Variables including gender, cirrhosis, tumor capsule, PT, ALB, and Child-Pugh show differences between the two institutions. In the overall cohort, the development cohort and the validation cohort, respectively, 136 patients (61.5\%), 82 patients $(62.6 \%)$, and 54 patients $(60 \%)$ had early recurrence $(<2$ years); 96 patients $(43.4 \%), 54$ patients $(41.2 \%)$, and 42 patients (46.7\%) had 6-month VER; 121 patients (54.8\%), 72 patients $(60.0 \%)$, and 49 patients $(54.4 \%)$ had 12 -month recurrence.

The comparison of clinicopathologic characteristics between patients with and without VER is summarized in Table 2. We found that people developed VER tend to have these clinical factors: the performance of major resection, lymph node dissection, tumor size $>=5 \mathrm{~cm}$, the presence of MiVI, MaVI, and SN, GGT> $=75 \mathrm{U} / \mathrm{L}$, $\mathrm{ALP}>=78 \mathrm{U} / \mathrm{L}, \mathrm{AFP}>=20 \mathrm{ng} / \mathrm{mL}, \mathrm{CA} 19-9>=25 \mathrm{U} /$ $\mathrm{mL}$, and $\mathrm{DCP}>=200 \mathrm{mAU} / \mathrm{mL}$.

\section{Construction and evaluation of the VER model}

The results of univariable logistic regression analysis and multi-variable logistic regression analysis of VER are listed in Table 3. The independent risk factors of VER including the presence of MiVI (odds ratio $3.73,95 \% \mathrm{CI}$ : 1.43-9.71, $P=0.007$ ), the presence of MaVI (odds ratio 5.75, 95\% CI: $1.73-19.04, P=0.004)$, and CA19-9 $>=25$ $\mathrm{U} / \mathrm{mL}$ (odds ratio $2.48,95 \% \mathrm{CI}$ : $1-6.13, P=0.049$ ) were used to construct the VER model (Table 3). A nomogram integrating the independent risk factors described above was constructed to facilitate the use of our predicting model of the VER (Fig. 2).

The C-indexes of the prediction of the VER model in development cohort and validation cohort were $0.77(95 \%$ CI: $0.69-0.85)$ and 0.76 (95\% CI: 0.66-0.86), while those of AJCC 8th staging was 0.64(95\% CI: $0.57-0.72)$ and 0.64(95\% CI: $0.54-0.74) . P$ value less than 0.05 showed the significant difference, which signified the superiority of VER model than AJCC 8th staging (Fig. 3A, B). In addition, the VER model showed a better net benefit than AJCC 8th staging in both cohorts (Fig. 4A, B). Calibration analysis also displayed a favorable agreement between the prediction and actual outcome of VER in both cohorts (Fig. 5A, B). Using a 50th centile of the VER risk score, 3.96 , in overall cohort, development cohort, and validation cohort, respectively, we stratified the patients into two risk groups with significant recurrence outcome $(P<$ 0.001) (Fig. 6A-C). 
Table 1 The clinicopathologic characteristics of patients in the development cohort and the validation cohort

\begin{tabular}{|c|c|c|c|}
\hline Variables & Development cohort $(n=131)$ & $\begin{array}{l}\text { Validation cohort } \\
(n=90)\end{array}$ & $P$ value \\
\hline Gender, male, $n(\%)$ & $117(89.3 \%)$ & $70(77.8 \%)$ & 0.032 \\
\hline Age [year, mean (IQR)] & $52.0[46.0,60.5]$ & $52.5[45.0,63.0]$ & 0.541 \\
\hline \multicolumn{4}{|l|}{ Hepatitis } \\
\hline NBNC, $n(\%)$ & $16(12.2 \%)$ & $14(15.6 \%)$ & 0.756 \\
\hline HBV, $n(\%)$ & $113(86.3 \%)$ & $75(83.3 \%)$ & \\
\hline HCV, $n(\%)$ & $2(1.5 \%)$ & $1(1.1 \%)$ & \\
\hline NAFLD, $n(\%)$ & $5(3.8 \%)$ & $6(6.7 \%)$ & 0.521 \\
\hline Cirrhosis, $n(\%)$ & $80.0(61.1 \%)$ & $69.0(76.7 \%)$ & 0.022 \\
\hline \multicolumn{4}{|l|}{ Resection type } \\
\hline Minor resection, $n(\%)$ & $44(33.6 \%)$ & $34(37.8 \%)$ & 0.619 \\
\hline Major resection, $n(\%)$ & $87(66.4 \%)$ & $56(62.2 \%)$ & \\
\hline Lymph node dissection, $n(\%)$ & $19(14.5 \%)$ & $21(23.3 \%)$ & 0.134 \\
\hline Tumor number, multiple, $n(\%)$ & $34(26.0 \%)$ & $25(27.8 \%)$ & 0.884 \\
\hline Tumor size [cm, mean (IQR)] & $5.10[3.60,7.35]$ & $5.30[3.03,9.00]$ & 0.737 \\
\hline Tumor capsule, present, $n(\%)$ & $85(64.9 \%)$ & $39(43.3 \%)$ & 0.002 \\
\hline Microvascular invasion, $n(\%)$ & $77(58.8 \%)$ & $64(71.1 \%)$ & 0.083 \\
\hline Macrovascular invasion, $n(\%)$ & $23(17.6 \%)$ & $31(34.4 \%)$ & 0.007 \\
\hline Satellite nodules, $n(\%)$ & $48(36.6 \%)$ & $28(31.1 \%)$ & 0.480 \\
\hline \multicolumn{4}{|l|}{ Edmondson-Steiner classification } \\
\hline$|/| \mid, n(\%)$ & $107(81.7 \%)$ & $67(74.4 \%)$ & 0.261 \\
\hline III/IV, n (\%) & $24(18.3 \%)$ & $23(25.6 \%)$ & \\
\hline $\mathrm{RBC}\left[10^{\wedge} 12 / \mathrm{L}\right.$, mean $\left.(\mathrm{IQR})\right]$ & $4.63[4.40,4.92]$ & $4.74[4.29,5.12]$ & 0.615 \\
\hline $\mathrm{Hb}[\mathrm{g} / \mathrm{L}$, mean $(\mathrm{IQR})]$ & $143[133,150]$ & $145[132,155]$ & 0.781 \\
\hline PLT[10^9/L, mean $(I Q R)]$ & $175[127,235]$ & $180[126,216]$ & 0.994 \\
\hline Neutrophil count[10^9/L, mean (IQR)] & $3.77[3.09,4.76]$ & $3.97[2.85,5.36]$ & 0.116 \\
\hline Lymphocyte count [10^9/L, mean (IQR)] & $1.59[1.16,1.92]$ & $1.42[1.15,1.83]$ & 0.705 \\
\hline NLR[ratio, mean (IQR)] & $2.42[1.74,3.45]$ & $2.53[1.66,3.78]$ & 0.227 \\
\hline AST[U/L, mean (IQR)] & $39.4[39.4,126]$ & $34.5[25.0,58.0]$ & 0.133 \\
\hline $\mathrm{PT}[\mathrm{s}$, mean $(\mathrm{IQR})]$ & $11.5[11.0,12.1]$ & $13.4[12.8,14.1]$ & $<0.001$ \\
\hline TB[umol/L, mean (IQR)] & $13.3[10.5,18.1]$ & $7.00[3.80,12.1]$ & 0.870 \\
\hline ALB[g/L, mean (IQR)] & $41.4[39.0,44.5]$ & $39.5[36.0,42.8]$ & $<0.001$ \\
\hline GGT[U/L, mean (IQR)] & $66.0[33.5,138]$ & $65.0[42.0,128]$ & 0.171 \\
\hline ALP[U/L, mean $(I Q R)]$ & $87.0[69.5,118]$ & $94.0[74.3,117]$ & 0.339 \\
\hline AFP[ng/mL, mean (IQR)] & $51.0[7.95,373]$ & $76.5[9.13,1540]$ & 0.250 \\
\hline CEA[ng/mL, mean (IQR)] & $2.50[1.70,3.70]$ & $2.86[1.70,4.40]$ & 0.725 \\
\hline CA19-9[U/mL, mean (IQR)] & $27.7[13.8,71.3]$ & $20.4[10.2,44.8]$ & 0.986 \\
\hline $\mathrm{DCP}[\mathrm{mAU} / \mathrm{mL}$, mean $(\mathrm{IQR})]$ & $64.0[23.0,412]$ & $110[40.0,940]$ & 0.057 \\
\hline Intraoperative blood loss [mL, mean (IQR)] & $200[100,300]$ & $200[100,288]$ & 0.105 \\
\hline Intraoperative blood transfusion, $n$ (\%) & $37(28.2 \%)$ & $33(36.7 \%)$ & 0.240 \\
\hline \multicolumn{4}{|l|}{ Child-Pugh } \\
\hline $\mathrm{A}, n(\%)$ & $130(99.2 \%)$ & $83(92.2 \%)$ & 0.018 \\
\hline B, $n(\%)$ & $1(0.8 \%)$ & $7(7.8 \%)$ & \\
\hline \multicolumn{4}{|l|}{ AJCC 8th } \\
\hline$|\mathrm{A}-| \mid, n(\%)$ & $111(84.7 \%)$ & $71(78.9 \%)$ & 0.347 \\
\hline III-IV, n (\%) & 20 (15.3\%) & $19(21.1 \%)$ & \\
\hline
\end{tabular}

Continuous variables were presented as number (percentage) and compared using the chi-square test or Fisher exact test. Normally distributed continuous variables were presented as mean (standard deviation, IQR). HBV hepatitis B virus, HCV hepatitis C virus, NAFLD non-alcoholic fatty liver disease, RBC red blood cell, $H b$ hemoglobin, PLT platelet count, NLR neutrophils/lymphocytes ratio, AST aspartate aminotransferase, $P T$ prothrombin time, $T B$ total bilirubin, $A L B$ albumin, GGT gamma-glutamyl transpeptidase, ALP alkaline phosphatase, AFP alpha-fetoprotein, CEA carcinoembryonic antigen, CA19-9 carbohydrate antigen 19-9, DCP decarboxylic prothrombin, AJCC American Joint Committee on Cancer 
Table 2 Comparison of clinicopathologic characteristics between patients with and without VER

\begin{tabular}{|c|c|c|c|}
\hline Variables & VER $(n=96)$ & Non-VER $(n=125)$ & $P$ value \\
\hline Gender(male) & 79 (82.3\%) & $108(86.4 \%)$ & 0.515 \\
\hline Age (>= 65 years) & $15(15.6 \%)$ & $24(19.2 \%)$ & 0.608 \\
\hline \multicolumn{4}{|l|}{ Hepatitis } \\
\hline NBNC, $n(\%)$ & $12(12.5 \%)$ & $18(14.4 \%)$ & 0.668 \\
\hline HBV, $n(\%)$ & $82(85.4 \%)$ & $106(84.8 \%)$ & \\
\hline HCV, n (\%) & $2(2.1 \%)$ & $1(0.8 \%)$ & \\
\hline NAFLD, $n(\%)$ & $5(5.2 \%)$ & $6(4.8 \%)$ & 1 \\
\hline Cirrhosis (yes) & $65(67.7 \%)$ & $84(67.2 \%)$ & 1 \\
\hline Resection type (major resection) & $70(72.9 \%)$ & $73(58.4 \%)$ & 0.036 \\
\hline Lymph node dissection (yes) & $24(25.0 \%)$ & $16(12.8 \%)$ & 0.031 \\
\hline Tumor number (multiple) & $30(31.3 \%)$ & $29(23.2 \%)$ & 0.235 \\
\hline Tumor size $(>=5 \mathrm{~cm})$ & $66(68.8 \%)$ & 55 (44.0\%) & $<0.001$ \\
\hline Tumor capsule (present) & $52(54.2 \%)$ & $72(57.6 \%)$ & 0.709 \\
\hline Microvascular invasion (present) & $77(80.2 \%)$ & $64(51.2 \%)$ & $<0.001$ \\
\hline Macrovascular invasion (present) & $38(39.6 \%)$ & $16(12.8 \%)$ & $<0.001$ \\
\hline Satellite nodules (present) & $48(50.0 \%)$ & $28(22.4 \%)$ & $<0.001$ \\
\hline Edmondson-Steiner classification (III/IV) & $21(21.9 \%)$ & $26(20.8 \%)$ & 0.978 \\
\hline $\operatorname{RBC}(>=4 \times 10 \wedge 12 / L)$ & $10(10.4 \%)$ & $13(10.4 \%)$ & 1 \\
\hline $\mathrm{Hb}\left(<120^{*} \mathrm{~g} / \mathrm{L}\right)$ & $7(7.3 \%)$ & $9(7.2 \%)$ & 1 \\
\hline $\mathrm{PLT}(<300 \times 10 \wedge 9 / \mathrm{L})$ & $86(89.6 \%)$ & $117(93.6 \%)$ & 0.404 \\
\hline Neutrophil count( $>=5 \times 10 \wedge 9 / \mathrm{L})$ & $63(65.6 \%)$ & $97(77.6 \%)$ & 0.068 \\
\hline Lymphocyte count $(>=1.5 \times 10 \wedge 9 / L)$ & $23(24.0 \%)$ & $32(25.6 \%)$ & 0.902 \\
\hline $\operatorname{NLR}(>=1.6)$ & $18(18.8 \%)$ & $30(24.0 \%)$ & 0.439 \\
\hline $\operatorname{AST}(>=40 \mathrm{U} / \mathrm{L})$ & $49(51.0 \%)$ & $56(44.8 \%)$ & 0.432 \\
\hline $\mathrm{PT}(>=13 \mathrm{~s})$ & 35 (36.5\%) & $40(32.0 \%)$ & 0.582 \\
\hline $\mathrm{TB}(>=17.1 \mathrm{umol} / \mathrm{L})$ & $22(22.9 \%)$ & $34(27.2 \%)$ & 0.569 \\
\hline $\mathrm{ALB}(<40 \mathrm{~g} / \mathrm{L})$ & $33(34.4 \%)$ & $48(38.4 \%)$ & 0.635 \\
\hline $\mathrm{GGT}(>=75 \mathrm{U} / \mathrm{L})$ & $54(56.3 \%)$ & $47(37.6 \%)$ & 0.009 \\
\hline $\operatorname{ALP}(>=78 \mathrm{U} / \mathrm{L})$ & $77(80.2 \%)$ & $72(57.6 \%)$ & $<0.001$ \\
\hline $\operatorname{AFP}(>=20 \mathrm{ng} / \mathrm{mL})$ & $72(75.0 \%)$ & $72(57.6 \%)$ & 0.011 \\
\hline $\mathrm{CEA}(>=5 \mathrm{ng} / \mathrm{mL})$ & $16(16.7 \%)$ & $18(14.4 \%)$ & 0.783 \\
\hline CA19-9(>= $25 \mathrm{U} / \mathrm{mL})$ & $63(65.6 \%)$ & $49(39.2 \%)$ & $<0.001$ \\
\hline $\mathrm{DCP}(>=200 \mathrm{mAU} / \mathrm{mL})$ & $41(42.7 \%)$ & $32(25.6 \%)$ & 0.011 \\
\hline Intraoperative blood loss(>= $200 \mathrm{~mL})$ & $60(62.5 \%)$ & $63(50.4 \%)$ & 0.097 \\
\hline Intraoperative blood transfusion (yes) & $28(29.2 \%)$ & $42(33.6 \%)$ & 0.578 \\
\hline
\end{tabular}

$H B V$ hepatitis $B$ virus, $H C V$ hepatitis C virus, NAFLD non-alcoholic fatty liver disease, $R B C$ red blood cell, $H b$ hemoglobin, PLT platelet count, NLR neutrophils/ lymphocytes ratio, $A S T$ aspartate aminotransferase, $P T$ prothrombin time, $T B$ total bilirubin, $A L B$ albumin, GGT gamma-glutamyl transpeptidase, $A L P$ alkaline phosphatase, AFP alpha-fetoprotein, CEA carcinoembryonic antigen, CA19-9 carbohydrate antigen 19-9, DCP decarboxylic prothrombin

\section{Discussion}

cHCC is a subtype of primary liver carcinoma composed of HCC and ICC, with a high incidence of recurrence [1-5]. Some researches display that patients with $\mathrm{cHCC}$ tend to have recurrence earlier than those with HCC, and similar to those with ICC $[2,3,9,10,24]$. Several previous studies reported that more than half of patients with cHCC suffered a dismal prognosis of 2-year early recurrence [4-8]. Similarly, 136 out of 221 patients (61.5\%) experienced early recurrence in our study. Therefore, concentrating on ER with a cut-off of 2 years may not be appropriate to the recognition of the recurrence highrisk patients.

In the field of other malignant tumors, previous studies have brought forward the definition of VER and researched risk factors of VER [11-13, 25]. Up to now, no one has proposed the definition of VER in CHCC. On the basis of our multiple center data, $43.4 \%$ of patients (96 out of 221) had recurrence within 6 months after the hepatic resection and $67.1 \%$ of those suffered the 
Table 3 Univariable logistic regression analysis and multivariable logistic regression analysis of VER in development cohort

\begin{tabular}{|c|c|c|c|c|}
\hline \multirow[t]{2}{*}{ Variables } & \multicolumn{2}{|c|}{ Univariable logistic regression analysis } & \multicolumn{2}{|c|}{$\begin{array}{l}\text { Multivariable logistic regression } \\
\text { analysis }\end{array}$} \\
\hline & OR $(95 \% \mathrm{Cl})$ & $P$ value & OR $(95 \% \mathrm{Cl})$ & $P$ value \\
\hline Gender(male) & 2.83(0.75-10.69) & 0.124 & & \\
\hline Age ( $>=65$ years) & $0.5(0.17-1.5)$ & 0.219 & & \\
\hline \multicolumn{5}{|l|}{ Hepatitis } \\
\hline HBV vs NBNC & $1.62(0.53-4.98)$ & 0.396 & & \\
\hline HCV vs NBNC & $2.2(0.11-42.74)$ & 0.602 & & \\
\hline NAFLD (yes) & $2.21(0.36-13.67)$ & 0.395 & & \\
\hline Cirrhosis (yes) & $1(0.49-2.05)$ & 0.993 & & \\
\hline Resection type (major resection) & $1.82(0.85-3.91)$ & 0.122 & & \\
\hline Lymph node dissection (yes) & $1.72(0.65-4.56)$ & 0.278 & & \\
\hline Tumor number (multiple) & $2.65(1.19-5.89)$ & 0.017 & $1.06(0.36-3.14)$ & 0.920 \\
\hline Tumor size $(>=5 \mathrm{~cm})$ & $1.94(0.95-3.94)$ & 0.069 & & \\
\hline Tumor capsule (present) & $0.76(0.37-1.56)$ & 0.449 & & \\
\hline Microvascular invasion (present) & $3.09(1.45-6.57)$ & 0.003 & $3.73(1.43-9.71)$ & 0.007 \\
\hline Macrovascular invasion (present) & $5.44(1.98-14.96)$ & 0.001 & $5.75(1.73-19.04)$ & 0.004 \\
\hline Satellite nodules (present) & $3.54(1.68-7.46)$ & $<0.001$ & $2.5(0.98-6.4)$ & 0.056 \\
\hline Edmondson-Steiner classification(III/IV) & $0.66(0.26-1.68)$ & 0.387 & & \\
\hline $\mathrm{RBC}(>=4 \times 10 \wedge 12 / \mathrm{L})$ & $0.8(0.22-2.88)$ & 0.733 & & \\
\hline $\mathrm{Hb}(<120 \times \mathrm{g} / \mathrm{L})$ & $1.97(0.42-9.2)$ & 0.387 & & \\
\hline $\operatorname{PLT}\left(<300 \times 10^{\wedge} 9 / \mathrm{L}\right)$ & $0.8(0.25-2.53)$ & 0.704 & & \\
\hline Neutrophil count $\left(>=5 \times 10^{\wedge} 9 / \mathrm{L}\right)$ & $0.57(0.26-1.29)$ & 0.181 & & \\
\hline Lymphocyte count $(>=1.5 \times 10 \wedge 9 / L)$ & $0.97(0.43-2.18)$ & 0.937 & & \\
\hline $\operatorname{NLR}(>=1.6)$ & $0.57(0.23-1.43)$ & 0.230 & & \\
\hline $\mathrm{AST}(>=40 \mathrm{U} / \mathrm{L})$ & $1(0.5-2.01)$ & 0.991 & & \\
\hline $\mathrm{PT}(>=13 \mathrm{~s})$ & $1.25(0.4-3.95)$ & 0.704 & & \\
\hline $\mathrm{TB}(>=17.1 \mathrm{umol} / \mathrm{L})$ & $0.83(0.39-1.75)$ & 0.618 & & \\
\hline $\mathrm{ALB}(<40 \mathrm{~g} / \mathrm{L})$ & $1.03(0.47-2.23)$ & 0.949 & & \\
\hline $\mathrm{GGT}(>=75 \mathrm{U} / \mathrm{L})$ & $1.76(0.87-3.55)$ & 0.116 & & \\
\hline $\operatorname{ALP}(>=78 \cup / L)$ & $4.07(1.79-9.23)$ & $<0.001$ & $2.03(0.77-5.33)$ & 0.152 \\
\hline $\operatorname{AFP}(>=20 \mathrm{ng} / \mathrm{mL})$ & $2.77(1.28-5.97)$ & 0.009 & $1.97(0.78-4.96)$ & 0.152 \\
\hline $\mathrm{CEA}(>=5 \mathrm{ng} / \mathrm{mL})$ & $0.84(0.29-2.46)$ & 0.747 & & \\
\hline CA19-9(>= $25 \mathrm{U} / \mathrm{mL})$ & $3.66(1.73-7.73)$ & $<0.001$ & $2.48(1-6.13)$ & 0.049 \\
\hline $\mathrm{DCP}(>=200 \mathrm{mAU} / \mathrm{mL})$ & $2.62(1.24-5.57)$ & 0.012 & $1.43(0.57-3.58)$ & 0.440 \\
\hline Intraoperative blood loss(>= $200 \mathrm{~mL})$ & $0.96(0.47-1.94)$ & 0.906 & & \\
\hline Intraoperative blood transfusion (yes) & $0.82(0.38-1.79)$ & 0.622 & & \\
\hline
\end{tabular}

$H B V$ hepatitis B virus, $H C V$ hepatitis C virus, NAFLD non-alcoholic fatty liver disease, $R B C$ red blood cell, $H b$ hemoglobin, $P L T$ platelet count, NLR neutrophils/ lymphocytes ratio, $A S T$ aspartate aminotransferase, $P T$ prothrombin time, $T B$ total bilirubin, $A L B$ albumin, GGT gamma-glutamyl transpeptidase, $A L P$ alkaline phosphatase, AFP alpha-fetoprotein, CEA carcinoembryonic antigen, CA19-9 carbohydrate antigen 19-9, DCP decarboxylic prothrombin, $C I$ confidence interval, $O R$ odds ratio

recurrence (96 out of 143) had recurrence within 6 months after the hepatic resection. Meanwhile, several previous studies proposed the definition of VER using a cut-off of 6 months in the research of HCC and ICC $[12,13]$. Given that $\mathrm{cHCC}$ consists of HCC and ICC, we deem it appropriate to determine the optimal cut-off of VER in cHCC as 6 months like most of the researches of HCC and ICC [11-13]. In addition, several researches demonstrated that patients with PLC who had VER suffered a significant lower OS than those who did not have VER $[11,13]$.

As such, this study concentrated on the recurrence happened within 6 months after the hepatic resection for patients with $\mathrm{cHCC}$. We identified the independent risk factors of VER and constructed a prediction model integrating MiVI, MaVI, and CA19-9 > $=25 \mathrm{U} / \mathrm{mL}$. Using the 
Points

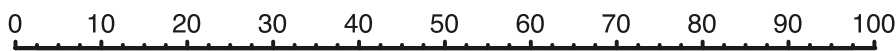

MiVIstatus

yes

no

MaVI

no

yes

CA199

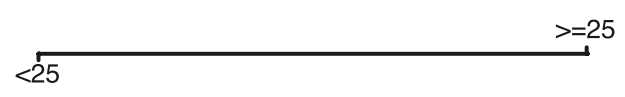

Total Points

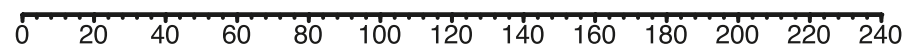

VER possibility

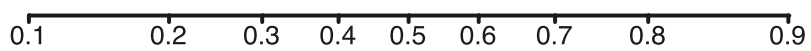

Fig. 2 VER nomogram integrating microvascular invasion, macrovascular invasion, and CA19-9> $=25 \mathrm{U} / \mathrm{mL}$

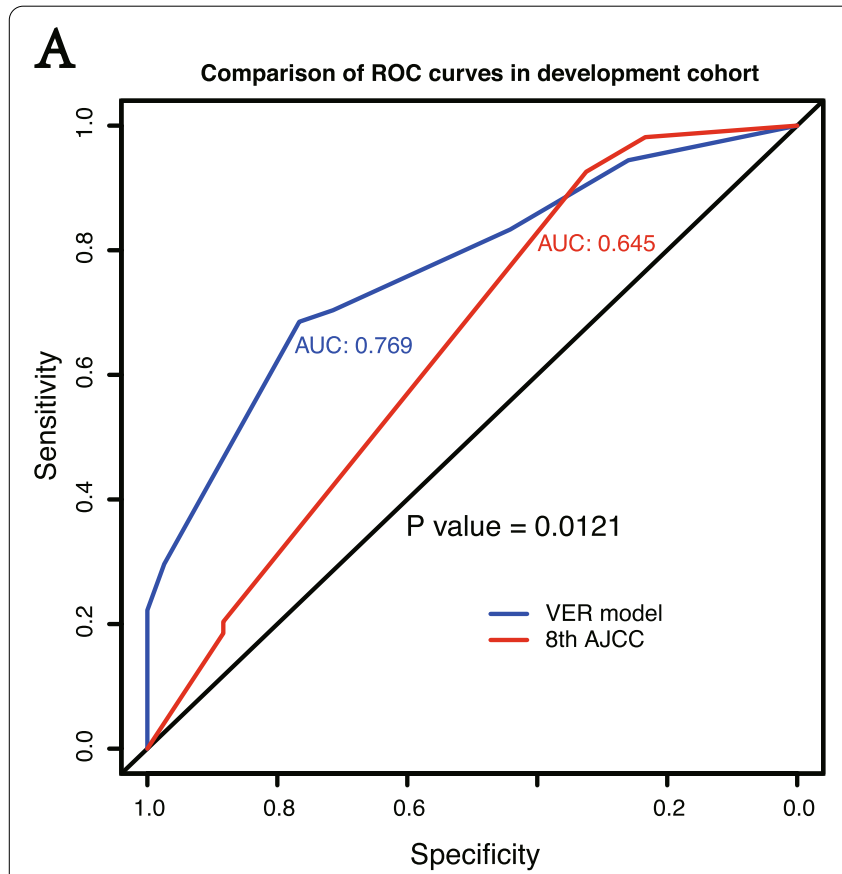

B

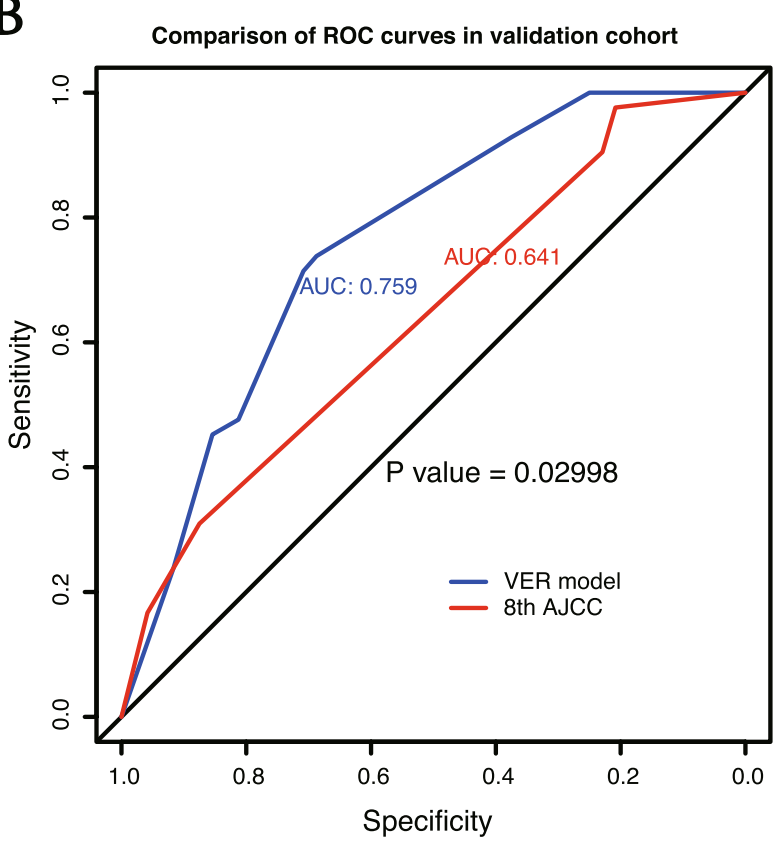

Fig. 3 ROC curves for VER model versus 8th AJCC in the development cohort $(P=0.012)(\mathbf{A})$ and the validation cohort $(P=0.030)(\mathbf{B})$. Our model shows better discrimination with C-indexes of 0.77 ( $95 \%$ Cl: $0.69-0.85$ ) and 0.76 (95\% Cl: 0.66-0.86) than AJCC 8th staging with C-indexes of 0.64 (95\% Cl: $0.57-0.72)$ and 0.64 (95\% Cl: $0.54-0.74)$ in the development cohort and validation cohort, respectively

VER model, we stratified all patients into two groups with significantly discrete risk of VER $(P<0.001)$. The high risk group consisting of $48.0 \%$ of all patients accounts for $71.9 \%$ of VER (Fig. 6A). The recognition of recurrence high-risk patients is of vital importance, because the clinician could suggest more vigorous surveillance strategy or appropriate anti-tumor strategies to them. To the best of our knowledge, our study is the first to define and predict VER in cHCC after hepatic resection. An easy-to-use website calculator and a nomogram were provided to facilitate the clinical use. 

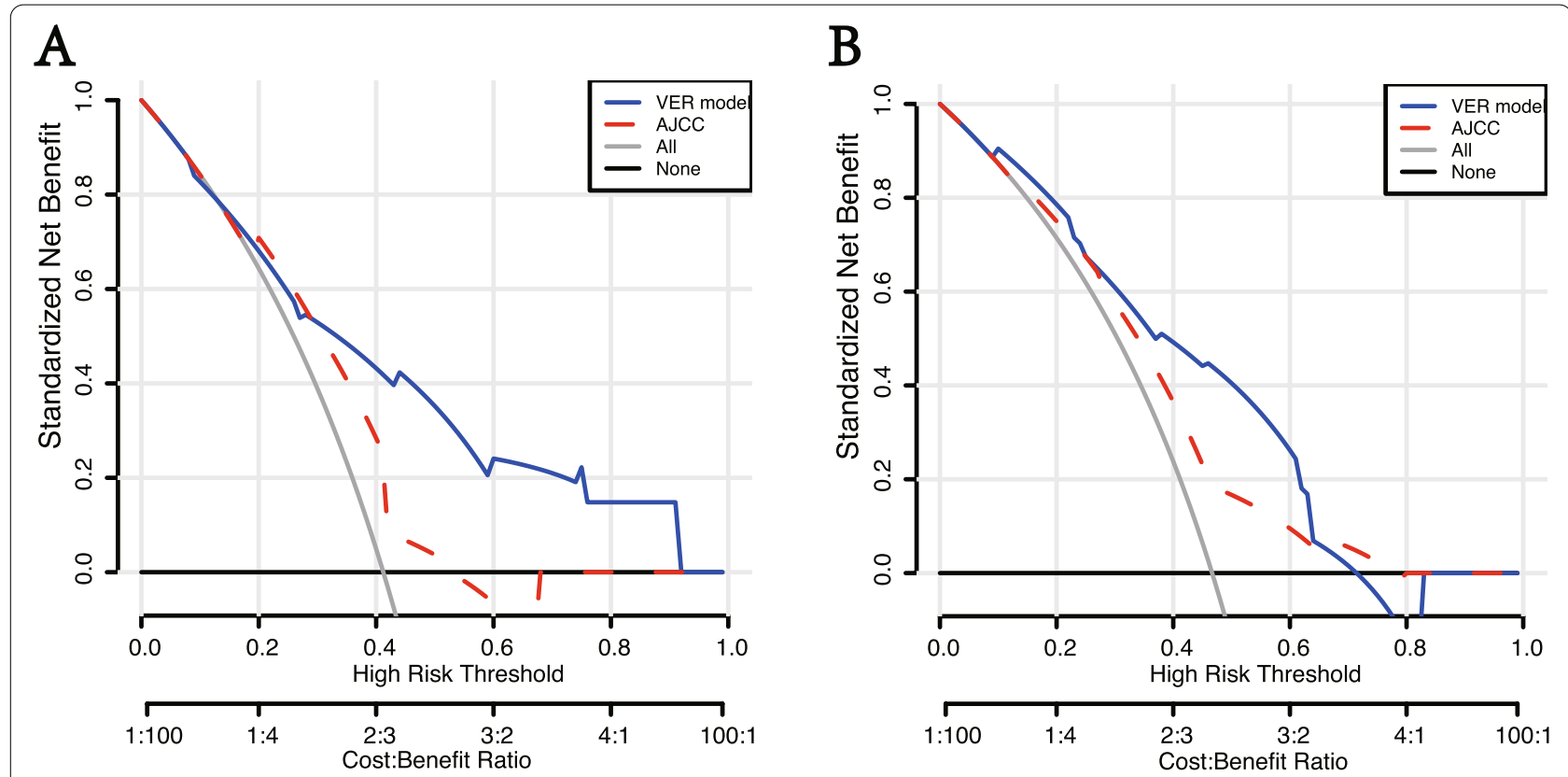

Fig. 4 Decision curve analysis for VER model versus 8th AJCC in the development cohort (A) and the validation cohort (B). Our VER model showed a better net benefit than AJCC 8th staging
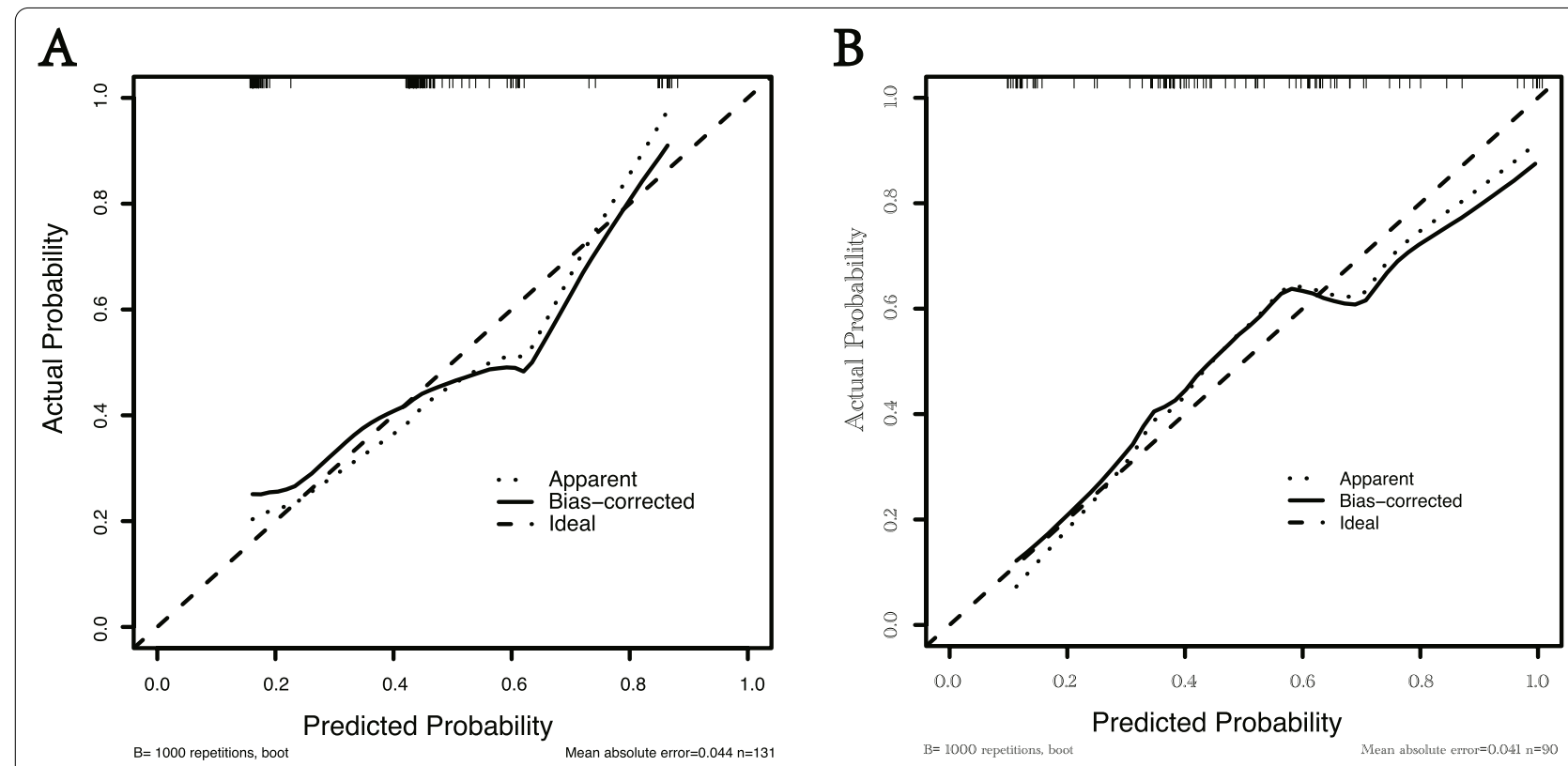

Fig. 5 Calibration analysis for VER model in the development cohort (A) and the validation cohort (B). Calibration analysis displayed a favorable agreement between the prediction and actual outcome of VER in both cohorts

The clinicopathological features of cHCC resemble those of HCC and ICC [3-5]. There is no existing predictive staging system that is commonly used for cHCC and applying the staging systems for $\mathrm{HCC}$ or ICC to $\mathrm{cHCC}$ may be problematic. From this perspective, we need a model to predict the recurrence or even VER for patients with cHCC. AJCC staging system is one of the traditional systems applied to the prediction of recurrence in primary liver carcinoma [20], many previous studies have demonstrated the predictive value of AJCC staging 


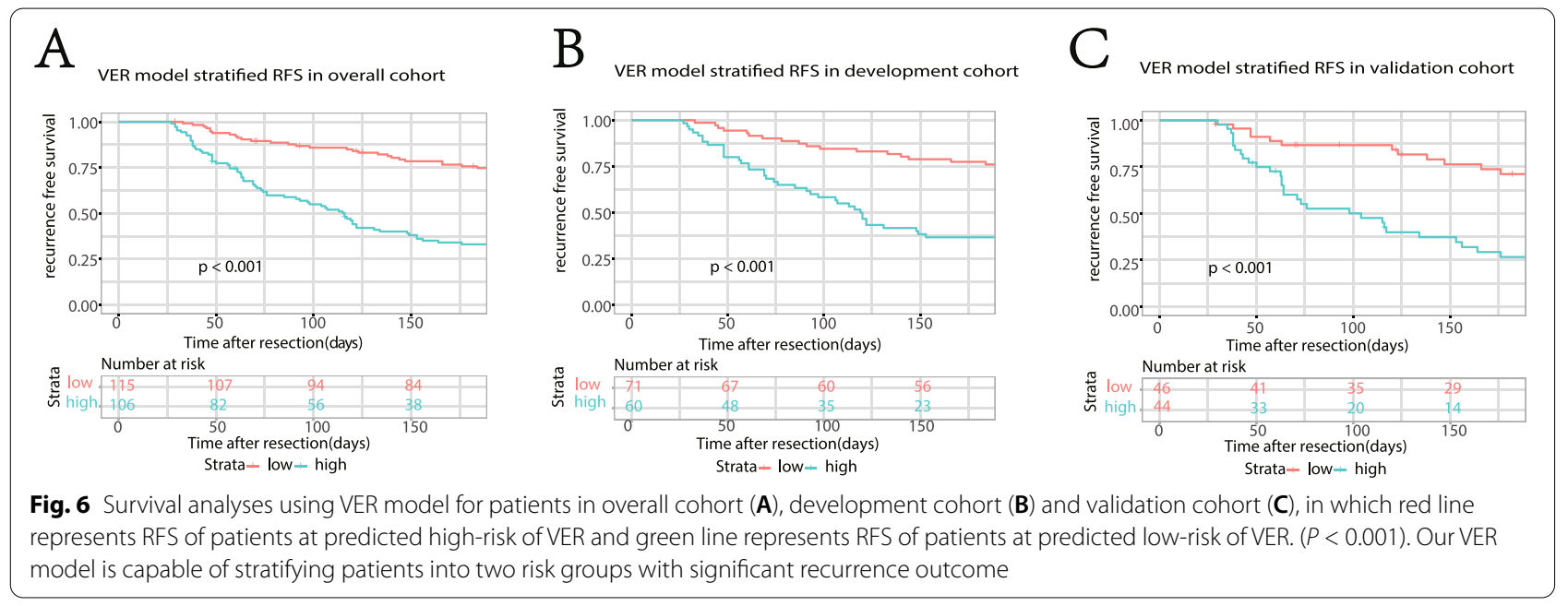

$[26,27]$. Nevertheless, ROC curves displayed the superior accuracy of our VER model than AJCC 8th staging according to higher $C$-indexes ( 0.77 vs 0.64 in development cohort $(P=0.012), 0.76$ vs 0.64 in validation cohort $(P=0.030)$ ) (Fig. 3A, B). Coincidentally, DCA analyze showed that our VER model provided obvious superior net benefit than AJCC 8th staging (Fig. 4A, B). Therefore, our VER model is more practical and more powerful than AJCC 8th staging in clinical use. In addition, the calibration analyze of our VER model also displayed favorable outcome.

Due to the rarity of $\mathrm{cHCC}$ and the variety of definitions and pathological types of cHCC, the research on $\mathrm{cHCC}$ and its prognosis has been tough and scant. Previous researches demonstrated that the independent risk factors for the recurrence of $\mathrm{cHCC}$ include tumor recurrence, tumor size ,metastases, age, MiVI, MaVI, SN, regional organ invasion, elevated CA19-9, ALP, and CEA as well as GGT [1-5]. A limited number of researches identified independent risk factors for the early recurrence of $\mathrm{cHCC}$ as follows: tumor size, tumor number, MiVI, MaVI, SN, lymph node metastasis, Midkine, DCP, CA19-9, and poor differentiation $[4-6,28]$. Additionally, some research suggest that because cHCC consists of mixed elements of both HCC and ICC, the risk factors for both HCC and ICC would be those for cHCC, which signifies the predictive value of the portion of HCC and ICC $[9,29,30]$. Multi-variable logistic regression analysis identified MiVI, MaVI, and CA199 as independent factors for VER in our study, which is generally consistent with the previous reports.

According to the studies in HCC, MiVI, MaVI, and $\mathrm{SN}$, the $\mathrm{HCC}$ related features, are related to the invasion behavior of HCC [17-19, 31-33]. It is widely acknowledged that MiVI, MaVI, and SN are independent risk factors for intrahepatic metastasis, recurrence and survival in HCC [17-19, 31-33]. In the studies in $\mathrm{cHCC}$ $[4,28,34,35]$, some previous researches also demonstrated the relationship between MiVI, MaVI, SN, and RFS, which is consistent with the studies in HCC. However, this view still remains controversial because some researches did not identified MiVI, MaVI, and SN as independent risk factors in their analysis $[5,6,29,36]$. The cause of this controversy can be listed as follows: firstly MiVI, MaVI, and SN were proposed and deeply studied in HCC, actually the pathological features of MiVI, MaVI, and SN in cHCC have not yet been completely investigated and the definitions have not been formulated neither. Unlike tumor size, tumor number, and serum tumor markers (AFP, DCP, and CA19-9), which are readily available and quantifiable, the definitions of MiVI, MaVI, and SN are various or unmentioned in the researches of $\mathrm{cHCC}$, and most researchers tend to define MiVI, MaVI, and SN in cHCC following the definitions in HCC. Secondly, the number of studies in the recurrence of cHCC is limited due to its own rarity and wide variety of pathological subtypes. Our research emphasized the predictive value of MiVI, MaVI for VER in $\mathrm{CHCC}$, thus promoting further researches to determine the pathological features and definitions of MiVI and MaVI in cHCC.

Previous studies elucidated that the elevation of CA19-9 reflects extensive tumor burden, which signifies biologically aggressive behavior, poor differentiation and greater tumor volume of ICC, and correlates with bleak prognosis in ICC [29, 37, 38]. Consistent with this conclusion, some researchers declared the elevation of CA19-9 to be an independent risk factor for RFS and OS in cHCC $[5,29]$. It is well known that CA19-9 is a biomarker for ICC components and the prognosis of ICC is worse than that of HCC $[2,37,38]$. Ideally, more portion 
of ICC lead to worse prognosis for $\mathrm{cHCC}$ patients, i.e., ICC dominance (defined as ICC components more than $50 \%$ or $80 \%$ pathologically according to different studies) is an independent risk factor for recurrence and survival in cHCC. However, some researchers identified that no relationship was found between ICC dominance and the poor prognosis in $\mathrm{cHCC}[5,39]$. Unfortunately, due to the lack of clinical data, we did not include this variable. More researches are needed to elucidate the relationship between ICC dominance and prognosis in CHCC.

Additionally, people developed VER tend to undergo major resection or lymph node dissection (Table 2), while logistics analysis showed no correlation between the performance of major resection or lymph node dissection and VER (Table 3). Consistent with our research in cHCC, some studies show that the performance of minor resection seems to improve the prognosis in patients with HCC, compared to that of major resection $[15,40]$, because major resection may influence the postoperative liver function. But no statistically significant difference was found in both DFS and OS in patients underwent major or minor resection [15, 40]. Meanwhile, some researchers recommend lymph node dissection for patients with cHCC, due to the high probability of lymph node metastasis. But no reliable research has been carried out to support the perspective [29, 41]. Compared to HCC and ICC, management of cHCC is not yet standardized, and the choice of surgical procedure still remains controversial. More large-sample research were needed to standardize the treatment of $\mathrm{cHCC}$.

There are three main limitations to our study. First, the clinical data were collected from two centers retrospectively and the sample size is limited, so the information bias, heterogeneity in patients' characteristics, and different surgical levels between two centers should be taken into consideration. Previous studies identified some variables, AFP, DCP, ALP, tumor size, and tumor number, as independent risk factors for the recurrence of $\mathrm{cHCC}$. Nevertheless, these variables of which the $P$ value is small in univariable or multi-variable analysis were identified uncorrelated with VER of cHCC in other research. We need more multi-institutional, large sample-sized, and prospective studies to verify the conclusion. Second, we only enrolled patients from China. Nearly $85 \%$ of the enrolled patients had been infected by HBV while few of them had HCV infection or NAFLD. We also excluded those who did not received curative hepatic resection or prior anti-tumor treatments. So, the generalizability of the conclusion is limited. Third, due to the lack of clinical data, we did not include the survival data or the variables which were missing over $10 \%$, such as HCC- or ICC-dominance.

\section{Conclusions}

Nearly half of patients underwent curative hepatic resection for $\mathrm{CHCC}$ experienced VER, resulting in a dismal prognosis. This study identified the presence of MiVI, MaVI, and CA19-9 > $25 \mathrm{mAU} / \mathrm{mL}$ as the independent risk factors for VER in cHCC patients and constructed a nomogram and an easy-to-use online calculator (https://chcrecurrence.shinyapps.io/myCHC VER2) to help recognize the VER high-risk patients who may be suggested more vigorous surveillance strategy or appropriate anti-tumor strategies.

\section{Acknowledgements \\ Not applicable.}

\section{Authors' contributions}

Conception and design: Yijun Wu and Jingfeng Liu. Administrative support: Jingfeng Liu, Weiping Zhou, and Yongyi Zeng. Collection of data: Yijun Wu and Hongzhi Liu. Analysis of data: Yijun Wu, Jianxing Zeng, and Yifan Chen. Manuscript writing: Yijun Wu. All authors read and approved the final manuscript.

Funding

This study was supported by Fuzhou Science and Technology Bureau Project (2020-WS-57) and Fuzhou Science and Technology Bureau project (2020-WS-92)

\section{Availability of data and materials}

The data that support the findings of this study are available from the corresponding author upon reasonable request.

\section{Declarations}

Ethics approval and consent to participate

This study was conducted in accordance with the ethical guideline of the 1975 Declaration of Helsinki and obtained approval from the Institutional Ethics Committee of the Mengchao Hepatobiliary Hospital of Fujian Medical University. Informed consent has been given to each participants enrolled in this study.

\section{Consent for publication}

Not applicable.

\section{Competing interests}

The authors declare that they have no competing interests.

\begin{abstract}
Author details
${ }^{1}$ Department of Hepatobiliary Surgery, Mengchao Hepatobiliary Hospital of Fujian Medical University, Fuzhou 350025, People's Republic of China. ${ }^{2}$ Shengli Clinical Medical College of Fujian Medical University, Fuzhoum, People's Republic of China. ${ }^{3}$ Department of Hepatobiliary Surgery, Eastern Hepatobiliary Surgery Hospital, Second Military Medical University, Shanghai 200438, People's Republic of China. ${ }^{4}$ Fujian Medical University Cancer Hospital \& Fujian Cancer Hospital, No. 420, Fuma Road, Fuzhou 350014, Fujian, People's Republic of China. ${ }^{5}$ The Big Data Institute of Southeast Hepatobiliary Health Information, Mengchao Hepatobiliary Hospital of Fujian Medical University, Fuzhou 350025, People's Republic of China.
\end{abstract}

Received: 23 November 2021 Accepted: 18 February 2022

Published online: 28 February 2022

\section{References \\ 1. Allen RA, Lisa JR. Combined liver cell and bile duct carcinoma. Am J Pathol. 1949;25(4):647-55.}


2. Lee J-H, Chung GE, Yu SJ, et al. Long-term prognosis of combined hepatocellular and cholangiocarcinoma after curative resection comparison with hepatocellular carcinoma and cholangiocarcinoma. J Clin Gastroenterol. 2011;45(1):69-75.

3. Yin X, Zhang B-H, Qiu S-J, et al. Combined hepatocellular carcinoma and cholangiocarcinoma: clinical features, treatment modalities, and prognosis. Ann Surg Oncol. 2012;19(9):2869-76.

4. Wang $T$, Yang $X$, Tang $H$, et al. Integrated nomograms to predict overall survival and recurrence-free survival in patients with combined hepatocellular cholangiocarcinoma (CHCC) after liver resection. Aging5. 2020;12(15):15334-58.

5. Yamashita Y-I, Aishima S, Nakao Y, et al. Clinicopathological characteristics of combined hepatocellular cholangiocarcinoma from the viewpoint of patient prognosis after hepatic resection: High rate of early recurrence and its predictors. Hepatol Res. 2020;50(7):863-70.

6. Ma M-C, Chen Y-J, Chiu T-J, et al. Positive expression of Midkine predicts early recurrence and poor prognosis of initially resectable combined hepatocellular cholangiocarcinoma. BMC Cancer. 2018;18(1):227.

7. Jeon SK, Joo I, Lee DH, et al. Combined hepatocellular cholangiocarcinoma: LI-RADS v2017 categorisation for differential diagnosis and prognostication on gadoxetic acid-enhanced MR imaging. Eur Radiol. 2019;29(1):373-82.

8. Wang X, Wang W, Ma X, et al. Combined hepatocellular-cholangiocarcinoma: which preoperative clinical data and conventional MRI characteristics have value for the prediction of microvascular invasion and clinical significance? Eur Radiol. 2020;30(10):5337-47.

9. Ishii T, Ito T, Sumiyoshi S, et al. Clinicopathological features and recurrence patterns of combined hepatocellular-cholangiocarcinoma. World J Surg Oncol. 2020;18(1):319

10. Deng Z, Jin Z, Qin Y, et al. Efficacy of the association liver partition and portal vein ligation for staged hepatectomy for the treatment of solitary huge hepatocellular carcinoma: a retrospective single-center study. World J Surg Oncol. 2021;19(1):95

11. Tsilimigras DI, Sahara K, Wu L, et al. Very early recurrence after liver resection for intrahepatic cholangiocarcinoma: considering alternative treatment approaches. JAMA Surg. 2020;155(9):823-31.

12. Park M-S, Lee K-W, Yi N-J, et al. Optimal tailored screening protocol after living donor liver transplantation for hepatocellular carcinoma. J Korean Med Sci. 2014;29(10):1360-6.

13. Hirokawa F, Hayashi M, Asakuma M, et al. Risk factors and patterns of early recurrence after curative hepatectomy for hepatocellular carcinoma. Surg Oncol. 2016;25(1):24-9.

14. Collins GS, Reitsma JB, Altman DG, et al. Transparent reporting of a multivariable prediction model for individual prognosis or diagnosis (TRIPOD): the TRIPOD statement. BMJ. 2015;350:97594.

15. Dahiya D, Wu T-J, Lee C-F, et al. Minor versus major hepatic resection for small hepatocellular carcinoma (HCC) in cirrhotic patients: a 20-year experience. Surgery. 2010;147(5):676-85.

16. Edmondson HA, Steiner PE. Primary carcinoma of the liver: a study of 100 cases among 48,900 necropsies. Cancer. 1954;7(3):462-503.

17. Sheng $X$, Ji Y, Ren G-P, et al. A standardized pathological proposal for evaluating microvascular invasion of hepatocellular carcinoma: a multicenter study by LCPGC. Hepatol Int. 2020;14(6):1034-47.

18. Chan AWH, Zhong J, Berhane S, et al. Development of pre and postoperative models to predict early recurrence of hepatocellular carcinoma after surgical resection. J Hepatol. 2018;69(6):1284-93.

19. Yang T, Lu J-H, Lau WY, et al. Perioperative blood transfusion does not influence recurrence-free and overall survivals after curative resection for hepatocellular carcinoma: A Propensity Score Matching Analysis. J Hepatol. 2016;64(3):583-93.

20. Chun YS, PawlikTM, Vauthey J-N. 8th Edition of the AJCC Cancer Staging Manual: Pancreas and Hepatobiliary Cancers. Ann Surg Oncol. 2018;25(4):845-7.

21. Takahashi S, Konishi M, Nakagohri T, et al. Short time to recurrence after hepatic resection correlates with poor prognosis in colorectal hepatic metastasis. Jpn J Clin Oncol. 2006;36(6):368-75.

22. lasonos A, Schrag D, Raj GV, et al. How to build and interpret a nomogram for cancer prognosis. J Clin Oncol. 2008;26(8):1364-70.

23. Vickers AJ, Elkin EB. Decision curve analysis: a novel method for evaluating prediction models. Med Decis Making. 2006;26(6):565-74.
24. Zhang XF, Beal EW, Bagante F, et al. Early versus late recurrence of intrahepatic cholangiocarcinoma after resection with curative intent. Br J Surg. 2018;105(7):848-56.

25. Jung SW, Kim D-S, Yu YD, et al. Risk factors for cancer recurrence or death within 6 months after liver resection in patients with colorectal cancer liver metastasis. Ann Surg Treat Res. 2016;90(5):257-64.

26. Jeong S, Cheng Q, Huang L, et al. Risk stratification system to predict recurrence of intrahepatic cholangiocarcinoma after hepatic resection. BMC Cancer. 2017;17(1):464.

27. Huang J, Liu F-C, Li L, et al. Nomograms to predict the long-time prognosis in patients with alpha-fetoprotein negative hepatocellular carcinoma following radical resection. Cancer Med. 2020;9(8):2791-802.

28. Tian M-X, Luo L-P, Liu W-R, et al. Development and validation of a prognostic score predicting recurrence in resected combined hepatocellular cholangiocarcinoma. Cancer Manag Res. 2019;11:5187-95.

29. Kim KH, Lee SG, Park EH, et al. Surgical treatments and prognoses of patients with combined hepatocellular carcinoma and cholangiocarcinoma. Ann Surg Oncol. 2009;16(3):623-9.

30. Schizas D, Mastoraki A, Routsi E, et al. Combined hepatocellular-cholangiocarcinoma: An update on epidemiology, classification, diagnosis and management. Hepatobiliary Pancreat Dis Int. 2020;19(6):515-23.

31. Arnaoutakis DJ, Mavros MN, Shen F, et al. Recurrence patterns and prognostic factors in patients with hepatocellular carcinoma in noncirrhotic liver: a multi-institutional analysis. Ann Surg Oncol. 2014;21(1):147-54.

32. Peng W, Li C, Zhang X, et al. The impact of thrombocytopenia on prognosis of HBV-related small hepatocellular carcinoma: a propensity score matching analysis. World J Surg Oncol. 2021;19(1):46.

33. Wang Y-C, Lee J-C, Wu T-H, et al. Improving outcomes of liver resection for hepatocellular carcinoma associated with portal vein tumor thrombosis over the evolving eras of treatment. World J Surg Oncol. 2021;19(1):313.

34. Chu K-J, Lu C-D, Dong H, et al. Hepatitis B virus-related combined hepatocellular-cholangiocarcinoma: clinicopathological and prognostic analysis of 390 cases. Eur J Gastroenterol Hepatol. 2014;26(2):192-9.

35. Lee SD, Park S-J, Han S-S, et al. Clinicopathological features and prognosis of combined hepatocellular carcinoma and cholangiocarcinoma after surgery. Hepatobiliary Pancreat Dis Int. 2014;13(6):594-601.

36. He C, Zhang Y, Cai Z, et al. Competing risk analyses of overall survival and cancer-specific survival in patients with combined hepatocellular cholangiocarcinoma after surgery. BMC Cancer. 2019;19(1):178.

37. Liu H, Qiu G, Hu F, et al. Fibrinogen/albumin ratio index is an independent predictor of recurrence-free survival in patients with intrahepatic cholangiocarcinoma following surgical resection. World J Surg Oncol. 2021;19(1):218.

38. Tang $Y$, Zhang T, Zhou X, et al. The preoperative prognostic value of the radiomics nomogram based on CT combined with machine learning in patients with intrahepatic cholangiocarcinoma. World J Surg Oncol. 2021;19(1):45.

39. Ariizumi S-I, Kotera Y, Katagiri S, et al. Combined hepatocellular-cholangiocarcinoma had poor outcomes after hepatectomy regardless of Allen and Lisa class or the predominance of intrahepatic cholangiocarcinoma cells within the tumor. Ann Surg Oncol. 2012;19(5):1628-36.

40. Lang BH-H, Poon RT-P, Fan S-T, et al. Perioperative and long-term outcome of major hepatic resection for small solitary hepatocellular carcinoma in patients with cirrhosis. Arch Surg. 2003;138(11):1207-13.

41. Beaufrère A, Calderaro J, Paradis V. Combined hepatocellular-cholangiocarcinoma: An update. J Hepatol. 2021;74(5):1212-24.

\section{Publisher's Note}

Springer Nature remains neutral with regard to jurisdictional claims in published maps and institutional affiliations. 